Original Article

\title{
An Epidemiological Study: Assessment of Symptomatic Osteoarthritis and its Management in General Population of Karachi with Joint Pain
}

Aisal Khan, Syed Muhammad Mustahsan, Quratulain Eraj, Hina Parvez, Ayesha Anwar, Munazza Ali, Sameen Iqbal, Urooj Fatima, Aiman Warsi, Tayyabah Moeen, Sundas Dastagir,

Fateeha Tariq, Rehan Shamim \& Hafiza Fatima Aziz

Dow University of Health Sciences, Sindh Medical College Campus

Corresponding Author: mustu198@gmail.com

\section{Abstract}

Object: To determine the frequency of symptomatic osteoarthritis in general population of Karachi presenting with joint pain. Introduction: People easily get tired due to heavy duty occupations to keep pace with the society. Arthralgia commonly called as "Joint pain" is one of the most frequently observed complain, especially in individuals aged 50 above. Methods: A cross-sectional study was conducted at Jinnah Postgraduate Medical Centre (JPMC) from $1^{\text {st }}$ November 2013 to $25^{\text {th }}$ November 2013. The data was collected from 316 patients presenting to the department of rheumatology and orthopedics with joint pain. A structured questionnaire was designed based on the clinical symptomatology of osteoarthritis. It was administered to the participants aged above 18 yrs. old. $17.7 \%$ of males and $82.3 \%$ of females participated in the study. Result: Out of 316 patients with joint pain, $71.2 \%(n=225)$ were diagnosed osteoarthritis on the basis of symptoms present with a high prevalence in females than males. The most common joints to be involved in osteoarthritis were found to be knee joint (90.2\%) and hip joint $(55.7 \%)$. The majority of the patients $(n=109)$ had an X-ray of their joint and $n=125$ was getting analgesics as an effective therapy for osteoarthritis. Conclusion: The frequency of osteoarthritis is increasing in Karachi with globalization due to high level occupational stress along with recreational activity stress and other contributive factors. Female predominance as compare to that of man is quite alarming that needs to be controlled for maintaining future quality of life and providing ease for daily activities.

\section{Keywords}

Knee Joint, Arthralgia, Osteoarthritis, Analgesics, Quality of life

\section{Introduction}

As the world is globalizing at the fastest rate in recent ages the burden of social and occupational adjustment on a human body is increasing with time. People easily get tired due to heavy duty occupations to keep pace with the society. Arthralgia commonly called as "Joint pain" is one of the most frequently observed complain, especially in individuals aged 50 above (Osteoarthritis,
2008). There can be variety of causes to this problem but in regards with the many studies done previously all over the world osteoarthritis has always been found to be the commonest cause of this reported problem. Osteoarthritis (OA), as has been defined is a heterogeneous degenerative disorder of joint cartilage remodeling most commonly presenting as joint pain bringing about biochemical lysis of cartilage matrix 
with subsequent chronic symptoms with prominent functional limitations and ultimately failure of a joint as an organ (Osteoarthritis, 2008). The disease has variable features and responses to treatment, the most important being empowering individuals with skills and relative knowledge so as to help them and increasing their morale. Self-management approach has proven to be the key feature in success of arthritis treatment (Arden E., et al. 2008). The degenerative process of the disease brings about a series of changes in the articular cartilage along with the complete joint that includes softening of matrix, ulceration, loss of cartilage with sclerosis and eburnation, and osteophytes and subchondral cysts formation (Osteoarthritis, 2008). Clinically, the disease presents as joint pain, limitation of movements, typical radiographic changes and limitations of daily activities. It is not always necessary that the degree of joint pain correlates well with the clinical findings showing variable penetrance and polygenecity of the disease (Meulenbelt, 2012). There are a variety of causes that can lead to osteoarthritis including hereditary transmission, secondary to trauma or excessive use, or due to any other underlying metabolic abnormality. It is therefore a disease difficult to diagnose and is mainly diagnosed clinically along with the radiological findings and is treated symptomatically mainly focusing the most ideally close rehabilitation that can be achieved so as to keep pace with the daily activities of life.

Scarce epidemiological data regarding osteoarthritis in our part of the world and its observed recent increase in frequency demanded a detailed search about its frequency and the factors associated with it. Though its clinical presentation correlates with many other joint diseases, signs and symptoms are still used as primary diagnostic criteria where laboratory investigations are not usually available due to financial issues. Targeting osteoarthritis in general population with joint pain and its relevant data could help up health practitioners in diagnosing and making a rational plan for the management and prevention of the disease to get the solution for of people in our community.

The aim of our study is focused on the frequency of osteoarthritis in general population of Karachi with joint pain and the relevant data related to it thus providing an urgent need of development of methods for preventing rapidly increasing musculoskeletal disorders that impair activities of daily life (ADL) and quality of life (QOL), which can help our health practitioners in making a rational plan for the managing, treating and controlling the disease and overcoming the expectations of the individuals in our community facing this problem.

The term arthritis covers more than 100 diseases with variable causes and clinical presentations (Clough J. D., 2006). Among these the two most common are osteoarthritis and rheumatoid arthritis due to different causes and with somewhat overlapping presentation. Therefore, it is very essential to identify the correct type of arthritis so that relative management steps can be taken for proper treatment (Petursdottir U., et al. 2009). Osteoarthritis is a chronic, heterogeneous, degenerative disorder of articular cartilage due to repetitive trauma and inadequate repair process. The disease varies in clinical presentation and symptoms. Joint pain of varying degrees is the most common associated symptom (Sarmiento, A., 2012). There are various risk factors including 
genetic, constitutional, and biochemical which in various proportions contribute to the development of the chronic degenerative process (Osteoarthritis, 2008). The prognosis varies among individuals due to various. Poor prognosis accounts for higher mortality rate (Agel, J., et al. 2003). Some of the main factors causing poor prognosis in the management process are old age, obesity, severe trauma, multiple joint involvement, and any underlying chronic metabolic disease such as diabetes (Bálint, G., et al. 1998). outcomes of osteoarthritis are variable among individuals as well for example some people only face chronic pain over years, some get dependent on prostheses and splints while some of them have so sever involvement that the get bed ridden due to joint deformities and limited mobility (Oberhause C., et al. 2013; Pouli N., et al. 2013). Outcomes of this disorder also depends upon patient compliance with management strategies (Campbell R., et al. 2001; Deyle G. D., et al. 2000; Beswick A. D., et al. 2013; Bálint, G., et al. 1998). Some patients prefer home remedies while others prefer to urgently attend their physicians and follow the told guidelines accordingly.

\section{Methodology}

A cross-sectional study was conducted from $1^{\text {st }}$ November 2013 to $25^{\text {th }}$ November 2013 at department of rheumatology and orthopedics, Jinnah Post Graduate Medical Center which is one of the leading public health sector located in Karachi south, covering patients coming from all 18 towns of Karachi. The sample size was calculated using a non-probability convenient sampling and collected from 316 candidates presenting with joint pain.

A self-designed questionnaire containing 30 close ended questions were designed in English and translated in Urdu for the ease of respondents and was administered after taking a proper informed verbal consent. The pilot study was conducted to assess the authenticity of questionnaire. The questionnaire included questions based on clinical symptomatology of osteoarthritis and rheumatoid arthritis and their laboratory tests and its management. The osteoarthritis was diagnosed using a diagnostic criteria based on symptoms most closely related to osteoarthritis which includes positivity for joint pain and any three of six symptoms including swelling, crepitus (crackling sound), osteophytes (bony outgrowths), aggravation of pain with activity, morning stiffness, lasting for less than an hour and restriction in moving joint with full range of motion. The independent variables were gender, age, literacy and occupation. The patients aged above 18 yrs. presenting with joint pain were included in the study and the patients who were addictive, having any chronic illness other than osteoarthritis were excluded from the study.

The significance of the data was determined and analyzed by using Statistical Package of Social Sciences software (SPSS, Version 20). Confidence interval of $95 \%$ was taken with margin of error $5 \%$ and p-value of 0.05 The descriptive analysis was performed and frequencies of dependent variables was taken out and cross tabs was done between gender and osteoarthritis and most recommended treatment with osteoarthritis.

Written informed consent was taken by all participants before interviewing about the joint pain. The participants filled the questionnaire on their will. The study was approved by the Ethical Review Board.

\section{Results}

A total of 316 patients with joint pain were enrolled in the study and analysis of data 
from an epidemiological survey of a population of $17.7 \%(n=56)$ men and $82.3 \%$ $(n=260)$ women were studied in all districts of Karachi. The mean age of patients presented to the OPD were 47.7 years \pm 12.21 as shown in table 1 and majority of participants were highly educated $(n=128$, $40.5 \%$ ). Out of 316 participants, $71.2 \%$ $(n=225)$ were diagnosed osteoarthritis on the basis of symptoms present. After adjusting the prognostic factors at $95 \%$ confidence level the osteoarthritis was significantly associated with knee joint (OR=2.309, 0.973-5.48) in comparison with wrist joint $(\mathrm{OR}=2.203,1.32-3.65), \quad$ hip joint $(\mathrm{OR}=0.865,0.59-1.26)$ and foot joint $(\mathrm{OR}=0.871,0.61-1.08)$. The osteoarthritis was prevalent in the males $(n=32)$ as well as in females $(n=193)$ as shown in fig. 1 and there was a significant difference between osteoarthritis and gender (chi square $=6.56$, $\mathrm{p}=0.01$ ).

The Multiple Response procedure was used to calculate the most common complain of the patients i.e. pain aggregate with the daily activities presenting with osteoarthritis using the dichotomous variables as shown in table 2.

Independent sample t-test was done to compare means of osteoarthritis with overweight and traumatic joints as shown in table 3. It was found that the osteoarthritis associated with overweight patients was $1.69 \pm 0.464$ and associated with trauma was $1.79 \pm 0.034$. Using the t-statistics the $\mathrm{p}=0.953$ for overweight and $\mathrm{p}=0.854$ for traumatic joints.

Out of 225 osteoarthritic patients, $n=109$ of patients have their $\mathrm{X}$-rays done, $\mathrm{n}=66$ have their Vit. D level checked, $n=79$ have their Ca level checked, $n=46$ have blood ESR levels and $n=32$ have their $\mathrm{Rf}$ factor done to confirm the diagnosis of osteoarthritis. Of them, $n=195$ patients were taking analgesic as an effective therapy and $n=24$ got the joint replacement therapy as shown in table 4.

\begin{tabular}{|l|c|c|c|c|c|}
\hline \multicolumn{7}{|c|}{ Table 1: Descriptive Statistics } \\
\hline & N & Minimum & Maximum & Mean & Std. Deviation \\
\hline age of respondent & 316 & 20 & 83 & 47.97 & 12.214 \\
\hline
\end{tabular}

Table 2: Symptoms Associated with Osteoarthritis Frequencies

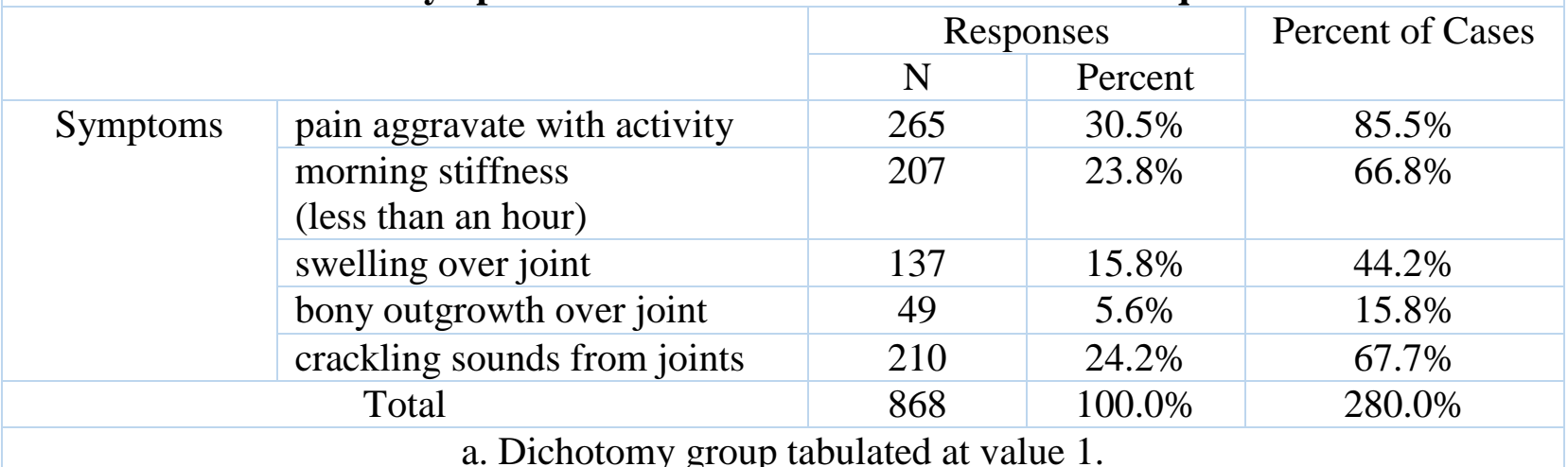




\begin{tabular}{|c|c|c|c|}
\hline \multicolumn{3}{|c|}{ Table 3: Osteoarthritis } \\
\hline Overweight & Mean & Std. Deviation & Std. Error Mean \\
\hline Traumatic Joint & 1.69 & .464 & .031 \\
\hline
\end{tabular}

Table 4: Independent Samples Test

Equal variances assumed

\begin{tabular}{|c|c|c|c|c|c|c|c|c|c|}
\hline \multicolumn{10}{|c|}{ Equal variances assumed } \\
\hline & \multicolumn{2}{|c|}{$\begin{array}{l}\text { Levene's Test } \\
\text { for equality of } \\
\text { variances }\end{array}$} & \multicolumn{7}{|c|}{ t-test for Equality of Means } \\
\hline & \multirow[t]{2}{*}{$\mathrm{F}$} & \multirow[t]{2}{*}{ Sig. } & \multirow[t]{2}{*}{$\mathrm{T}$} & \multirow[t]{2}{*}{ df } & \multirow[t]{2}{*}{$\begin{array}{l}\text { Sig. } \\
(2- \\
\text { tailed })\end{array}$} & \multirow[t]{2}{*}{$\begin{array}{c}\text { Mean } \\
\text { Differ } \\
\text { ence }\end{array}$} & \multirow[t]{2}{*}{$\begin{array}{c}\text { Std. Error } \\
\text { Differenc } \\
\text { e }\end{array}$} & \multicolumn{2}{|c|}{$\begin{array}{l}95 \% \text { Confidence } \\
\text { Interval of the } \\
\text { Difference }\end{array}$} \\
\hline & & & & & & & & Lower & Upper \\
\hline Overweight & .014 & .905 & -.059 & 166.537 & .953 & -.003 & .058 & -.117 & .110 \\
\hline $\begin{array}{l}\text { Traumatic } \\
\text { Joint }\end{array}$ & .656 & .418 & -.184 & 175.830 & .854 & -.011 & .060 & -.130 & .108 \\
\hline
\end{tabular}

Figure 1: Distribution of Osteoarthritis Among Gender

250

200

150

100

50

0

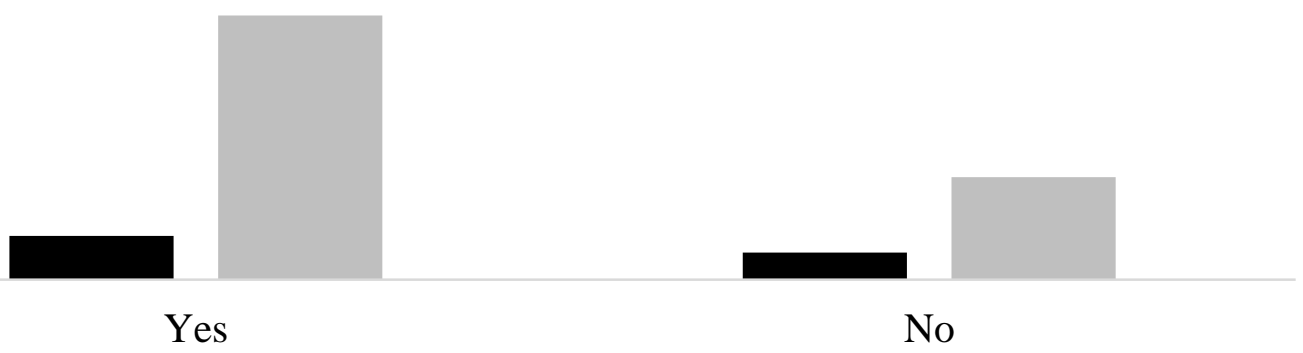

Male Female

\begin{tabular}{|l|l|c|c|c|}
\hline \multicolumn{1}{|c|}{ Table 5: } & \multicolumn{2}{c|}{ Osteoarthritis } & \multirow{2}{*}{ Total } \\
\cline { 2 - 4 } Treatment & Yes & No & \\
\hline \multirow{3}{*}{ Taking Analgesics } & 195 & 68 & 263 \\
\hline & Taking Vit D/ Ca Supplements & 118 & 38 & 156 \\
\hline & Doctor Advised Physiotherapy & 103 & 28 & 131 \\
\hline & Massage or Hot Packing at Home & 99 & 26 & 125 \\
\hline & Reducing Weight, If Over Wt & 70 & 28 & 98 \\
\hline & Doctor Advised to Use Splints/Braces/Insoles & 22 & 8 & 30 \\
\hline & Joint Replacement Therapy Advised & 24 & 1 & 25 \\
\hline
\end{tabular}




\section{Discussion}

\section{Joint Pain and Arthritis}

Arthritis is a term used for inflammation of one or more arthrosis or joints. This term covers a wide range of pathologies affecting joints some of which are osteoarthritis, rheumatoid arthritis, gouty arthritis, seronegative spondyloarthritis, and many more. The two most common among these are osteoarthritis and rheumatoid arthritis. The symptom of joint pain is commonly associated with the complaint of arthritis in varying forms depending upon which type of arthritis is present and either it is primary or secondary. Joint pain is usually the first presenting symptom in patients suffering from arthritis because it is very unusual to have no pain in an inflamed joint. Therefore, varying degrees of joint pain from mild, moderate to severe is usually present. In addition to this, patients may complain of pain in muscles, tendons, and bursae signifying "rheumatism" commonly known as pain in muscles and tendons (Clough $\mathrm{J}$. D., 2006). The burden of arthritis around the globe is on the rise due to lifestyle practices at present but it is not always a serious life threatening condition. Arthritis needs great attention because of the pain accompanying the problem. In some cases, it is the presenting feature of the disease causing it to occur such as carcinoma lung, SLE, hepatitis, T.B, AIDS, and rheumatic fever etc. therefore, it is very essential to make a correct diagnosis and treating the underlying pathology offers the best possible management to the disease which is putting such a great impact on the quality of life and affecting daily activities of individuals suffering from it (Clough J. D., 2006).

Three most important factors while making a diagnosis to differentiate variable types of arthritis are:
1.Assessing which and how many of the joints are affected either symmetrically or asymmetrically.

2.Over what period the joint has been involved either gradual or sudden and the frequency of joint involvement.

3.The intensity of associated pain, swelling, tenderness and other such factors along with other systemic signs such as skin and hair loss, ulcers, nodules, fever etc. (Clough J. D., 2006).

A skilled medical practitioner can easily conclude by assessing these signs and symptoms to what is going on but it is better to supplement the final diagnosis with radiological modalities and laboratory findings so as diagnosing incorrectly by any chance and not to further delay the correct diagnosis to take early management steps further. During analysis, total frequency of OA was found to be $71.2 \%$ of which the highest frequency of joint pain was found to be in knee joint $(90.2 \%)$ followed by hip $(55.7 \%)$, foot $(45.3 \%)$, and wrist $(44.6 \%)$ while $32.9 \%$ were having pain in all joints. According to W.H.O $40 \%$ of patients aged above 70 years are suffering from knee osteoarthritis and $25 \%$ of world's population is facing difficulties in daily activities having sever impacts of quality of life due to restricted joint movements caused by the development of osteoarthritis. (Agel, J., et al. 2003)

\section{Risk Factors of Osteoarthritis}

As we previously discussed, osteoarthritis is a complex disorder involving joints with multiple risk factors which can be widely divided into three major types:

1.Genetic causes: Inheritable causes are more in hand, knee, and hip although the 
cause of this or any responsible genes are for the most part unknown. (Preedy, V. R. 2010; Henrotin, Y., 2012).

2.Constitutional causes: These factors include age, sex, obesity, and high bone density etc.

3.Biochemical risk factors: These include any form of joint trauma, excessive occupational/recreational usage, joint laxity, mal-alignment, and reduced strength of muscles (Osteoarthritis, 2008).

Most of the causative burden of osteoarthritis has ever come from life style and environmental risk factors and are reversible by the modification of lifestyle or avoidable as in case of occupation and recreation still, individual risk factors vary and differs at different joint sites and the relative risk of occurrence of $\mathrm{OA}$ to that of its progression and outcome is also different. During analysis of the collected data we found that the disorder rises in frequency with age, peaks at 48 and thereafter the graph falls in frequency in Karachi.

Female gender has a higher frequency of osteoarthritis occurrence than the male gender and most of them were housewives working with inappropriate postures or excessive burden on joint due to activities which increases the risk of OA. We also found that $43 \%$ of the individual have a family member affected with the same condition and $31 \%$ of the individuals were advised to lose weight by their physicians. Development of trauma induced OA was found in $24.7 \%$. By this we can easily interpret that majority of the burden rising in Karachi is due to occupational and recreational activities with female predominance and other risk factors second this burden.

\section{Structural Pathology, Symptoms, Diagnostic and Prognostic Outcomes of Osteoarthritis}

As we discussed previously osteoarthritis is the degenerative process involving joint cartilage with variable causes and symptoms. The structural pathology of osteoarthritis can be best viewed radiographically even when the disease is minor presenting without any symptoms. It is only late in the disease that external deformities become visible (Osteoarthritis, 2008; Conaghan, et al. 2012; Conaghan, et al. 2009).

The structural alterations in cartilage occur due to various factors in combination and not due to a single reason like varying gait and weight with age apply variable patterns of burden on joints causing them to mould accordingly with continuous attenuation and loss of cartilage and as the person ages the body's regenerative capacity weakens progressively in accordance with the genetic makeover of a person with less regeneration after every induced trauma even at the cellular level (Moskowitz R. W., et al. 2007). The symptomatic presentation as we discussed previously doesn't always coincide with the radiological findings and is quite variable among individuals. Usually, $\mathrm{OA}$ is accompanied by deep achy pain, tenderness over joints, swelling, gelling, crackling sounds, morning stiffness of less than 30 minutes, and bony out growths over joints i.e. the osteophytes. (Rossky, E., 2000).

Patients often complain pain in all joints depending upon the cause along with difficulty in walking and getting up from chair or while offering prayers due to reduced range of motion because of pain and stiffness. The distinguishing factor between osteoarthritis and rheumatoid arthritis is 
mainly the aggravation of pain and stiffness in osteoarthritis while fading of these two symptoms in rheumatoid arthritis with activity (Clough J. D, 2006).

The prognostic outcomes of osteoarthritis are different for different joints being much better for hand osteoarthritis than for knee and hip among which hip has the worst prognostic outcome mostly requiring hip replacement surgery (Osteoarthritis, 2008). The disorder is commonly predominantly occurring in older individuals and is commonly associated with other factors in its cause such as diabetes, cardiovascular diseases, obesity very often, and psychosocial problems which all determines the final outcome of the disease. Some factors associated with poor prognosis include older age, varus deformity, overweight, multiple joints involvement and patient reluctance to treatment (Clough J. D, 2006, Day C. S., et al. 2003). One more important factor that we found during our study was the relationship between OA prognosis, literacy of individuals, and socioeconomic factors. The area we collected our data from was mostly attended by people with low level in literacy and low socio-economic scale. Therefore, we found that most of them who didn't have much idea about the disease, its progression, severity, and outcomes were more prone to adverse prognosis due to reluctant behavior with the management process and lacking of diagnostic data with them. This is one of the major causes of increasing osteoarthritis burden in this region (Campbell R., 2001). $32.9 \%$ of individuals were found to have pain in all joints which as discussed previously is one of the major causes of poor prognosis in osteoarthritis. A very low ratio of patients was advised laboratory testing of calcium \& vitamin D levels, ESR levels, and $\mathrm{RF}$ factor values along with X-rays. This shows most of them were diagnosed on the basis of clinical symptoms.

\section{Impact of Osteoarthritis on Individual's Activities and Social Life}

As the disorder has been found to be the most common cause of disability around the globe, it has significant impacts on daily activities and social life of an individual. The most common problematic complain among patients is that of pain which restricts their activities and cause them depression and cutoff from social life. Many of these individuals face persistent pain and presents mainly with this complaint unaware of the fact that they are suffering from any sort of arthritic disorder. Other than this involvement of large joints restricts patients' mobility and they face problems while walking, dressing, sitting, getting up, driving, and other such activities. The last and worst impact is that there is increased mortality rate with osteoarthritis in older individuals due to serious disabilities and poor prognostic factors including fractures of hip arthritic joint. (Iqbal M. N., et al. 2011). Societal impacts include heavy burden of cost expenditures for the management of this chronic disease to improve the daily activities and quality of life of patients with osteoarthritis. The disorder was estimated to be eighth leading cause of non-fatal disease burden and sixth leading cause of disability around the globe in 2003 (Osteoarthritis, 2008). Therefore, it has one of the world's largest economy burden rates. $82 \%$ and $73.7 \%$ were found to have difficulty in walking and getting up from chair respectively causing poor impact on performing daily activities and thus disturbing their quality of life.

\section{Management of Osteoarthritis}

Management of osteoarthritis is based on the severity of the disease. Pain and quality of 
life management is the key aspect in management strategies. (Coleman, S., et al 2008; Hasset G., 2002). There are various ways to control pain and to control the degenerative process so as to improve life quality and expectancy. (Dubin, A. et al. 2009). Pain killers including acetaminophen and NSAIDs like aspirin are frequently used in the management of OA while to slow the degenerative process as maximum as we can DMARDs are new modality use for this treatment step and their efficacy has been well in prognostic outcomes (Bradely J. D. 2013; Asokan GV., et al. 2011; Tizani A. 2010). Their doses and combination is adjusted in such a way so as to minimize the risk of side effects as close as possible. Other management steps include OA friendly exercises, hot packing massage, use of local muscle relaxants, physiotherapy and in last the joint replacement surgery being the mainstay of treatment when patient's condition is deteriorating every possible medical management (Fortin P. R., et al. 2002). Patients complaining of mild pain and difficulty in walking are advised to use external prostheses or splints for better support and posture. (Brandt, K. D, 2000). Our result shows that $7.9 \%$ of the individuals were advised joint replacement surgery which is a significant value when analyzing severity of the disease. According to result evaluation it was found that $83.2 \%$ patients were taking analgesics on doctors' advice signifying the pain control management strategies in the region of Karachi along with patient preferences. Physiotherapy clinics are being attended by $41.5 \%$ and $39.6 \%$ prefer home remedies such as hot packing and massage for pain relief.

\section{Conclusion}

In this study we found that the burden of osteoarthritis is on the rise in the region of
Karachi with significant impact on health and economical sectors due to rapidly deteriorating environment for the disease and high cost requires for its relative management which ultimately leads individuals to avoid treatment due to low socio economic and literacy status. This chronic degenerating disease is significantly affecting the quality of life and social environment around individuals making them even more prone to disabilities in regards of physical and mental health. (Brotzman, S. B., et al. 2011). Therefore, there is definitely an urgent need for developing such management modalities that will be easier and more practical to carry out despite the limitations of economy, patient coordination, and environmental control.

\section{Conflict of Interest}

There is no conflict of interest

\section{Acknowledgment}

On behalf of all the authors, we would like to thank Dr. Kiran Mehtab (Head of Department of Community Medicine) for her kind support, concerned behavior and guidance in every step.

\section{References}

- Agel, J., Akesson, K., Amadio, P. C., Anderson, M., Badley, E., Balint, G., ... Yoshizawa, H. (2003). The burden of musculoskeletal conditions at the start of the new millennium. World Health Organization - Technical Report Series, (919).

- Arden E., Arden N., Hunter D., "Osteoarthritis." New York, Oxford University Press Inc., (2008) p. 3-8.

- Asokan GV., Hussain M. S., Hussain A., and Al-Safwan Z. AM., "Osteoarthritis Among Women in Bahrain: A Public 
Health Audit." Oman Medical Journal, Oman U.A.E, (2011).

- Bálint, G., Szebenyi, B., \& Kirwan, J. (1998). The use of guidelines for managing and treating osteoarthritis. Disease Management and Health Outcomes, 3(3), 131-142.

- Beswick A. D., Wylde V., Hill R. G., "What proportion of patients report longterm pain after total hip or knee replacement for osteoarthritis? A systematic review of prospective studies in unselected patients." School of Clinical Sciences, University of Bristol, Bristol, UK, Clinical Education Research Group, Peninsula Medical School, Plymouth, UK, (2013).

- Bradely J. D., Brandt K. D., Katz B. P., "Comparison of an anti-inflammatory dose of ibuprofen, an analgesic dose of ibuprofen and acetaminophen in the treatment of patients with osteoarthritis of knee." The New England Journal of Medicine, Massachusetts Medical Society. (2013).

- Brandt, K. D. "Diagnosis and nonsurgical management of osteoarthritis, (2nd ed.). "Professional Communications, Inc., Caddo, OK, (2000).

- Brotzman, S. B., Manske, R. C., \& Daugherty, K. "Clinical orthopaedic rehabilitation: an evidence-based approach (3rd ed.)." Elsevier Mosby, Philadelphia. (2011).

- Campbell R., Evans M., Tucker M., "Why don't patients do their exercises? Understanding non-compliance with physiotherapy in patients with osteoarthritis of the knee." Journal of Epidemiology and Community Health, U.S.A, (2001).

- Clough J. D. "Arthritis: A Cleveland Clinical Guide." The Cleveland Clinical
Foundation, the Cleveland Clinical Press, (2006): p-1-3.

- Coleman, S., Briffa, N. K., Carroll, G., Inderjeeth, C., Cook, N., \& McQuade, J. (2008). Effects of self-management, education and specific exercises, delivered by health professionals, in patients with osteoarthritis of the knee. BMC Musculoskeletal Disorders, 9(1), 1.

- Conaghan, P., \& Nelson, A. E. "Osteoarthritis (2nd ed.)." Abingdon: Health Press, (2012).

- Conaghan, P., \& Sharma, L. (2009). "Fast facts: osteoarthritis." Health Press, Abingdon, Oxford, UK, (2009).

- Day C. S., Gelberman R., Patel A. A, "Basal joint osteoarthritis of the thumb: a prospective trial of steroid injection and splinting." The American Journal of Hand Surgery, U.S.A, (2003).

- Deyle G. D., Henderson N. E., Matekel R. L., "Effectiveness of Manual Physical Therapy and Exercise in Osteoarthritis of the Knee: A Randomized, Controlled Trial." American College of Physicians, Fort Sam Houston, Texas; and Madigan Army Medical Center, Tacoma, Washington, (2000).

- Dubin, A., Pilitsis, J., Argoff, C. E., \& McCleane, G. (2009). Pain Management Secrets. Elsevier Health Sciences.

- Fortin P. R., Penrod J. R., Clarke A. E., "Timing of total joint replacement affects clinical outcomes among patients with osteoarthritis of the hip or knee." The American College of Rheumatology, U.S.A, (2002).

- Hassett, G. "Osteoarthritis." Medicine, King's College London, (2002). P- 4043.

- Henrotin, Y. "Nonpharmacological therapies in the management of osteoarthritis." Bentham Books, Saif Zone, Sharjah, U.A.E, (2012). 
- Iqbal M. N., Haidri F. R., Motiani B., "Frequency of factors associated with knee osteoarthritis." JPMA, Liaquat National Hospital, J.P.M.C, Karachi, (2011).

- Meulenbelt "Osteoarthritis and cartilage." The Netherlands. Elsevier Ltd. Leiden University Medical Center, 2300 RC Leiden, (2012): pp. 218-222.

- Moskowitz R. W., Altman R. D., Hochberg M. C. "Osteoarthritis: Diagnosis and Medical/surgical Management $4^{\text {th }} \quad$ Ed." $\quad$ Lippincott Williams and Wilkins, U.S.A, (2007); p 3-31.

- National Collaborating Center for Chronic Conditions. "Osteoarthritis: National clinical guideline for care and management in adults. London: Royal College of Physicians of London; (2008) P. 13-19.

- Oberhause C., Escorpizo R., Boonen A., "Statistical validation of the brief International Classification of Functioning, Disability and Health Core Set for osteoarthritis based on a large international sample of patients with osteoarthritis." American College of Rheumatology, U.S.A, (2013).

- Petursdottir A. and Halldorsdottir S., "Facilitators and
Barriers to Exercising Among People With Osteoarthritis: A Phenomenological Study." Journal of American physiotherapy, Oslo, Norway, (2009).

- Pouli N., Nair R. D., Lincoln N. B., "The experience of living with knee osteoarthritis: exploring illness and treatment beliefs through thematic analysis." Disability and Rehabilitation, An Internal Multidisciplinary Journal, Arthritis Research UK Pain Centre, University of Nottingham, Nottingham, UK, (2013).

- Preedy, V. R., \& Watson, R. R "Handbook of disease burdens and quality of life measures." Springer, New York, (2010).

- Rossky, E. "Protection of arthritic joints: a manual for use by occupational therapists and patients." Dept. of Occupational Therapy, Moss Rehabilitation Hosp. Philadelphia, (2000).

- Sarmiento, A. "Orthopedics: seeking a balance." Jaypee Brothers Medical Pub., New Dehli, (2012).

- Tizani A., "Harvard's nursing guide to drugs." Elsevier, Elsevier Australia, Victoria Avenue, Chatswood, (2010); p 228-235. 